\title{
Fiber Bundle Segmentation Using Spectral Embedding and Supervised Learning
}

\author{
Dorothée Vercruysse, Daan Christiaens, Frederik Maes, Stefan Sunaert, and \\ Paul Suetens
}

\begin{abstract}
Diffusion-weighted imaging and tractography offer a unique approach to probe the microarchitecture of brain tissue noninvasively. Whole brain tractography, however, produces an unstructured set of fiber trajectories, whereas clinical applications often demand targeted tracking of specific bundles. This work presents a novel, hybrid approach to fiber bundle segmentation, using spectral embedding and supervised learning. Training data of 20 healthy subjects is labeled with a parcellation-based method, and used to train support vector machine and random forest classifiers. Cross-validation was used to avoid overfitting. Results on testing data of 5 independent subjects show a clear improvement over unsupervised methods. Moreover, estimating the label probabilities allows to reduce the effect of outliers.
\end{abstract}

\section{Introduction}

Diffusion-weighted magnetic resonance imaging (DWI) [1] is a unique approach to probe the microarchitecture of brain tissue noninvasively. Based on measurements of the local diffusion anisotropy, and assuming that diffusion is larger along the direction of neuronal fibers [3], numerous methods exist to estimate the local fiber orientation distribution function (fODF) [7, 17]. Tractography methods aim to reconstruct white matter (WM) pathways, usually by following the estimated fiber directions. Two distinct groups of stepwise tractography methods exist: deterministic methods, which

D. Vercruysse · D. Christiaens · F. Maes · P. Suetens

Medical Image Computing, Dept. of Electrical Engineering, KU Leuven, Belgium;

Medical Imaging Research Center, University Hospitals Leuven, Belgium;

iMinds, Medical IT Department, Belgium

e-mail: daan.christiaens@esat.kuleuven.be

S. Sunaert

Translational MRI, Dept. of Imaging \& Pathology, KU Leuven, Belgium;

Medical Imaging Research Center, University Hospitals Leuven, Belgium 
select the most likely track direction in every step [2,5,12], and probabilistic methods, which draw random samples from the fODF or a similar distribution $[15,18]$.

Whole brain tractography, however, produces an unstructured set of fiber trajectories, whereas clinical applications often demand targeted tracking of specific bundles. Generally, this requires manual delineation of regions-of-interest (ROI), which is tedious and subjective work. Therefore, a number of automated bundle segmentation methods have been presented, which can be categorized into fiber clustering methods and parcellation-based methods [13]. Fiber clustering methods aim to group neighbouring or similar tracks into clusters, which can then be assigned anatomically meaningful labels from an atlas $[10,11,14]$. Parcellation-based methods, on the other hand, employ carefully defined ROIs in atlas space, e.g., a cortical parcellation, to segment and label predefined WM bundles [21]. While this allows for highly specific labeling, tracts that do not intersect the ROIs are excluded from the analysis and may hence result in a low sensitivity.

This work presents a novel, hybrid approach to fiber bundle segmentation, using spectral embedding and supervised learning. The white matter query language (WMQL) [21], a parcellation-based method, is used to generate ground-truth labels to train the classifiers. The sensitivity and specificity of the proposed method is calculated using cross-validation, and results on unlabeled, whole brain tractography data are presented.

\section{Methods and Materials}

The proposed method is based on spectral embedding, a dimensionality reduction technique that allows to represent each track as a vector in the embedding space such that nearby tracks (provided some distance metric), are mapped onto nearby embedding vectors. O'Donnell et al. [14] were the first to use spectral embedding for fiber clustering, using the unsupervised $\mathrm{k}$-means algorithm in the embedding space. Instead, we propose to use supervised learning, specifically support vector machines (SVM) and random forests, on the embedding vectors. The training data, required for this approach, is provided by the WMQL.

\subsection{Spectral Embedding}

The aim of spectral embedding is to find a meaningful representation of the input data in a lower dimensional space. When used for clustering, this representation should preferably group data points that score high for a certain similarity measure. As such, similarity relationships are represented spatially, i.e., every fiber is represented as a point and nearby points generally correspond to similar trajectories, which simplifies the clustering [14]. 
Assuming that fibers following a similar trajectory belong to the same anatomical bundle, a pairwise fiber affinity $w_{i j}$ between tracks $i$ and $j$ is calculated from the symmetrized mean closest point (MCP) distance $d_{i j}=d_{j i}=\left(\mathrm{MCP}_{i j}+\mathrm{MCP}_{j i}\right) / 2$ via a Gaussian kernel, i.e., $w_{i j}=e^{-\frac{d_{i j}^{2}}{\sigma^{2}}}$. These affinities are computed between all pairs of fibers, resulting in a symmetric affinity matrix $\mathbf{W}$. Based on the Normalized Cuts algorithm by Shi and Malik [16], the embedding vectors are then computed using the eigenvectors $\mathbf{U}$ of the normalized affinity matrix $\mathscr{W}=\mathbf{D}^{-\frac{1}{2}} \mathbf{W} \mathbf{D}^{-\frac{1}{2}}$, where $\mathbf{D}$ is a diagonal matrix containing the row sums of $\mathbf{W}$.

In practice, the entire affinity matrix is too large to compute. Therefore, as described in Fowlkes et al. [8], its eigenvectors are approximated using the Nyström method [8]. Instead of calculating all pairwise fiber affinities, a random subset of tracks is chosen (containing an equal amount of fiber trajectories from each subject) and only the affinities from all subjects to this subset are computed. The layout of the entire affinity matrix is shown in Fig. 1. Submatrix A contains the pairwise affinities between the fibers of the subset and $\mathbf{B}$ those of the rest of the fibers to the subset. The largest submatrix $\mathbf{C}$ does not have to be calculated, reducing the computation time considerably.

Note that in order to make a multi-subject atlas, spectral clustering needs to be performed in all subjects together. Therefore, a registration step is required to transform the tracks to a common space, e.g., MNI space, using subject dependent deformation fields. As such, the fibers of all subject are treated as if they originate from one brain for training, which results in one embedding space.

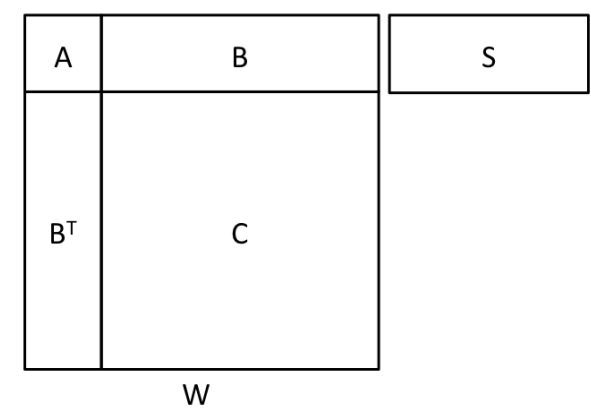

Fig. 1 Block structure of the normalized affinity matrix $\mathscr{W}$, used in the Nyström method. The regions in submatrices $\mathbf{A}$ and $\mathbf{B}$ denote the individual subjects. Submatrix $\mathbf{C}$ represents the part that does not need to be calculated when using the Nyström method. For the automatic segmentation of a new subject, matrix $\mathbf{S}$ contains the affinity values between the new tracks and the subset of matrix A. (reproduced from [14]) 


\subsection{Supervised Classification}

The proposition made is to cluster the embedding vectors into anatomically relevant bundles using supervised clustering instead of k-means. The training of the classifiers is performed on labeled WMQL output tracks. The WMQL is a parcellation based segmentation method developed by Wassermann et al. [21] which uses queries based on 1) anatomical terms that state if a fiber tract traverses or ends in a certain anatomical region of the brain, 2) relative position terms that state if a tract lies, for instance, lateral or frontal of a certain anatomical structure such as the amygdala and 3) logical operations that are for example conjunctions, disjunctions or exclusions of the previous two types of terms.

The supervised learning methods used in this study are support vector machines (SVM) and random forests. The basic principle of SVMs is to find a unique hyperplane by maximizing the margin between two classes [20]. Two different kernels are tested, linear SVMs and Gaussian radial basis function (RBF) kernels. The advantage of SVMs is that they are effective in high dimensional spaces. Furthermore, they employ only a subset of training points, the support vectors, in the decision function, which makes them memory efficient [6]. A drawback, however, is the slow training for large data sets. Random forests on the other hand, are classifiers developed by Breiman [4] which use the ensemble (forest) of decision trees, generated from random input vectors and using only a random subset of features for splitting nodes. Since the number of randomly selected features is much smaller than the number of input vectors, random forests learn fast on large datasets.

\subsection{Automatic Segmentation}

By training the classifiers, a white matter atlas is created which can be used for the automatic segmentation of fiber bundles from novel subjects. First, the new tracks must be registered or transformed to the atlas (MNI) space. Secondly, the affinity between the tracks of the new subject and the atlas is calculated. As illustrated in Fig. 1, a matrix $\mathbf{S}$ is calculated that contains the affinity values between the new tracks and the subset of tracks used for calculating $\mathbf{A}$. Note that $\mathbf{S}$ also has to be normalized [14]. Next, each new fiber will be embedded in the same embedding space as created for the original clustering. A detailed description of the eigenvector estimation of the novel subject is given by O'Donnell et al. [14]. The final step is the automatic segmentation itself, i.e., the cluster information from the atlas is applied to the new embedding vectors. In order to do this, the novel embedding vectors are used as input for the trained classifiers. 


\subsection{Materials}

Data of 25 healthy subjects were provided by the Human Conectome Project (HCP), WU-Minn consortium [19]. DWI data consists of $3 \times 90$ gradient directions at $b$ values 1000, 2000, and $3000 \mathrm{~s} / \mathrm{mm}^{2}$ and 18 non-diffusion weighted images $(b=0)$, at an isotropic voxel size of $1.25 \mathrm{~mm}$, and was corrected for motion and EPI distortions as described in [9]. The fODF, and subsequent deterministic and probabilistic fiber tracks (50000 each), were reconstructed with MRtrix [17] using default parameters. The tracks were segmented into 44 WM bundles using Wasserman's WMQL and the WM parcellation available in the HCP data set, resulting in approximately 4500 labeled tracks for each subject. All tracks are warped to MNI space using the deformation fields provided by the HCP. The multisubject deterministic and probabilistic training sets to create the atlases consist of the labeled tracks of 20 subjects, the other 5 form the test set. For affinity calculation $\sigma$ is set to $60 \mathrm{~mm}$. The submatrix A contains a random sample of 4000 tracks.

\section{Validation}

The proposed method was applied on the 20 training subjects, constructing the embedding space and training the SMV and random forest classifiers. To avoid overfitting, 5-fold cross-validation is used for the training. The true positive rate, calculated on the test set of 5 novel subjects and assuming their WMQL labels as ground truth, is given in Table 1. For comparison, k-means is included, using 200 clusters (identical to [14]), and assigning labels based on majority voting.

The scores of the supervised classifiers are all in the same range of around $95 \%$, with a maximum of $95.78 \%$ for the SVM with RBF-kernel on deterministic tracks. The true positive rate (TPR) for k-means, on the other hand, is below $85 \%$, hence the proposed method achieves over $10 \%$ improvement.

To evaluate the performance of the models on finding each individual bundle, scores are determined following a one-versus-the-rest principle. The performance plots in Fig. 2 give an overview of the results on deterministic tracks. Results are preferably expected in the upper left quadrant of the plot, where the TPR (sensitivity) is high and the false positive rate (FPR, $(1-$ specificity)) is low. Since the negative class will always be very large (43 bundles, versus 1 bundle in the positive class), true negatives $(\mathrm{TN})$ will be very high in comparison to false positives (FP). Therefore, the specificity will be high and the FPR low. Note that the $x$-axis in the plots only ranges from 0 till 0.025 instead of 1 , to give a more detailed view. The sensitivity is spread over a wider range. The green bundle that scores very low with every supervised classifier (Fig. 2a-c), represents the right superior occipitofrontal fasciculus, a bundle that contains very little to no tracks in most subjects. This explains the bad training performance. The plot for the k-means classifier (Fig. 2d) shows an overall larger spread, as was expected from the lower total clustering performance. 
Table 1 Validation scores (true positive rate, TPR)

\begin{tabular}{lcc}
\hline & TPR (deterministic) & TPR (probabilistic) \\
\hline K-means $(\mathrm{K}=200)$ & $84.40 \%$ & $81.97 \%$ \\
SVM, kernel=linear & $95.30 \%$ & $95.09 \%$ \\
SVM, kernel=RBF & $95.78 \%$ & $95.63 \%$ \\
Random forests & $95.07 \%$ & $94.77 \%$ \\
\hline
\end{tabular}

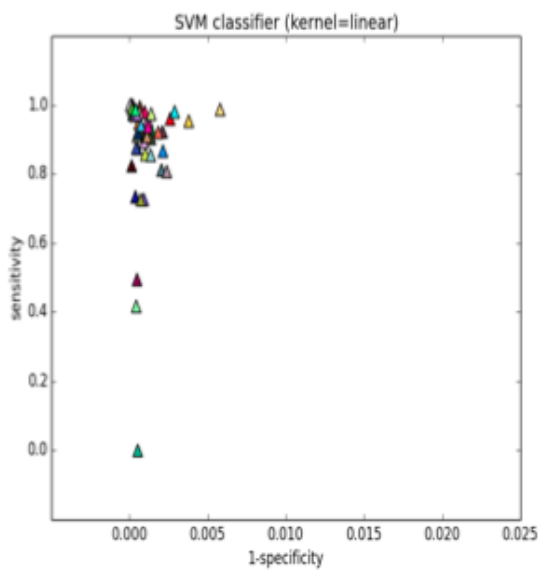

(a)

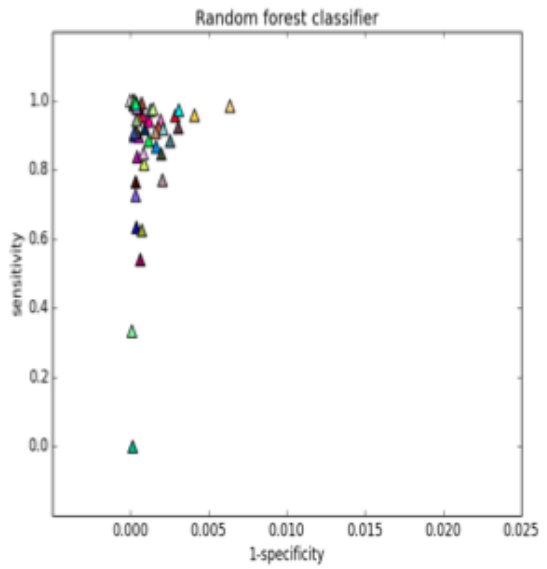

(c)

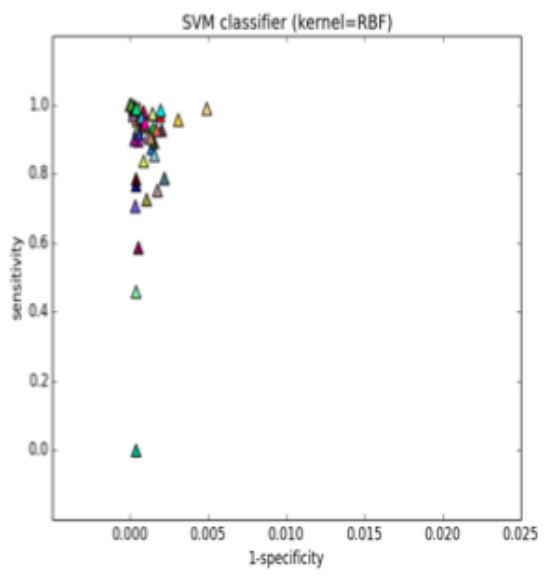

(b)

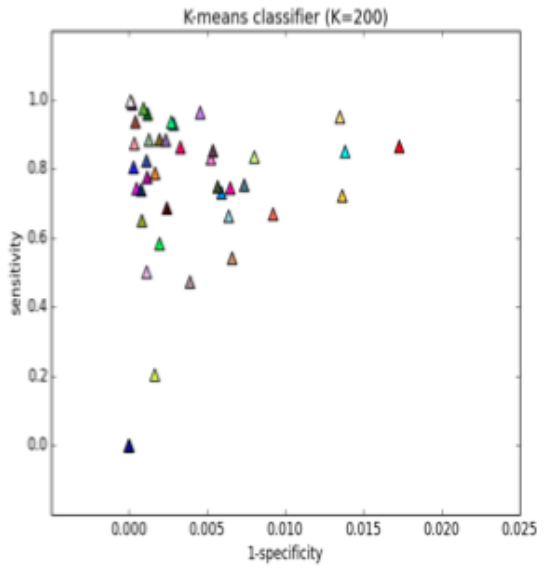

(d)

Fig. 2 Performance plots of the different classifiers for all 44 bundles (one-versus-the-rest) on deterministic WMQL output tracks, representing the true positive rate (sensitivity) versus the false positive rate $(1-$ specificity). The marker colors represent the bundle number. Note that the $x$-axis ranges from 0 to 0.025 to give a more detailed view. 


\section{Results}

The unsupervised and supervised classifiers that were trained as discussed above are now evaluated on the unlabeled whole brain track sets (deterministic and probabilistic, each containing 50000 tracks) of the test subjects. The tracks are warped to atlas space and projected onto the embedding space of the training data. Afterwards, their labels are estimated by using the resulting embedding vectors as input for the trained classifiers. In case of the k-means classifier, each track receives the label of the closest centroid in embedding space.

Since the whole track set is used as input, including many spurious tracks (i.e., false positives) and interrupted tracks that were not labeled by the WMQL, it is no longer possible to use the labels generated by the WMQL as ground truth to calculate, e.g., the TPR. Therefore, the results can only be analyzed visually by comparing the resulting segmentation with the WMQL output bundles, and with basic neuroanatomical knowledge.

First, the results are studied without considering the label probability, i.e., using maximum-likelihood classification. This is shown in Figs. 3 and 4 for deterministic and probabilistic tractography respectively, in a single subject. The top row shows the segmentation of the corticospinal tract (CST) in 1 subject. Notice the misclassified cerebellum (which is not defined in the WMQL queries) tracks in the k-means and linear SVM segmentation. Additionally, all classifiers label sagittal dispersions in the corona radiata as CST. The segmentation of the cingulum bundle (CB) contains misclassified tracks of the fornix (which is not labeled in the WMQL) and dispersing tracks from the corpus callosum.

In Fig. 5, a threshold is put on the labeling probabilities of the SVM with RBFkernel and the random forest classifier, in order to try to ameliorate the results by excluding outlier tracks. For the CST, increasing the threshold results in the exclusion of the corona radiata tracks, while the cut off tracks of the left CST bundle are still correctly labeled, contrary to the WMQL segmentation. The CB segmentation contains a lot less dispersions into the $\mathrm{CC}$ with a high threshold and also the tracks from the fornix are eliminated.

\section{Discussion}

The high scores for validation on labeled WMQL output tracks show the potential of the proposed method for fiber bundle segmentation. The true positive rates (TPR) for the supervised classifiers are considerably higher than that obtained by the k-means classifier. The performance evaluation across individual bundles allows to conclude that the results are in the same range for most bundles, except for three of them which contain little to no tracks in the training set. This could be solved by re-evaluating the WMQL queries for these specific bundles.

As expected, the results on complete whole brain tractography data sets, which include all outlier tracks (i.e., false positives), are not as good as those on validation 

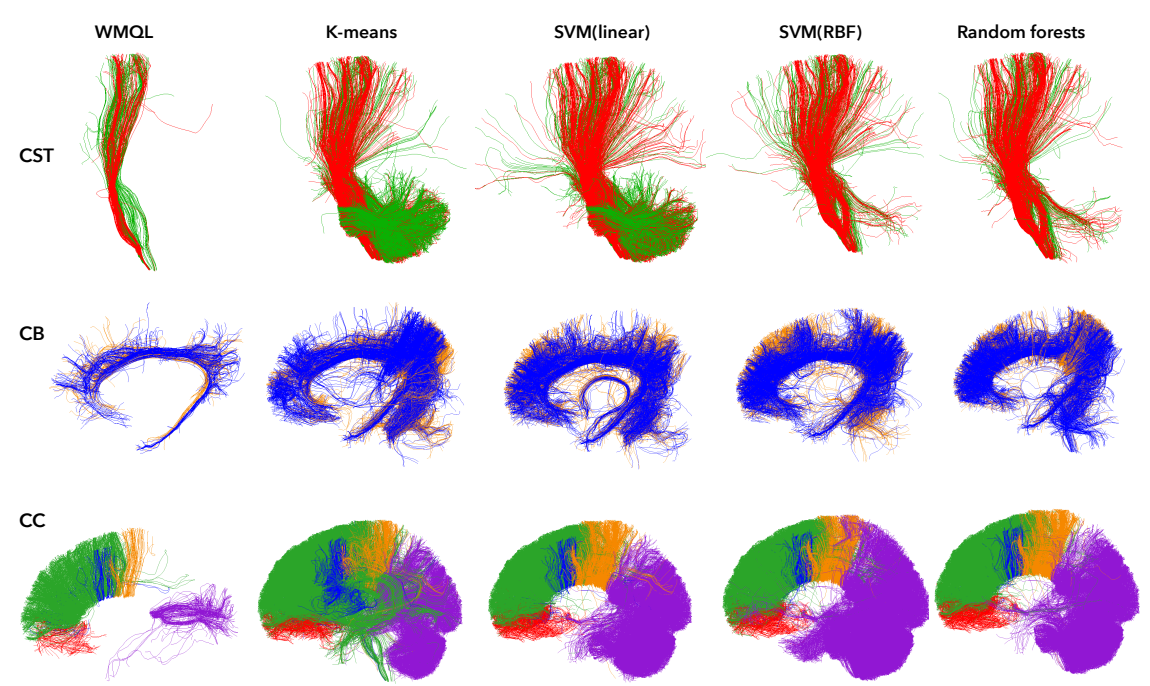

AF
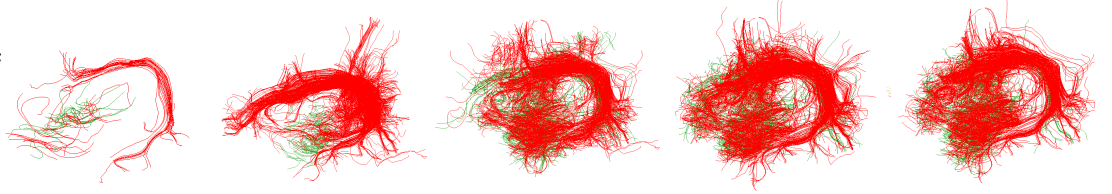

UF
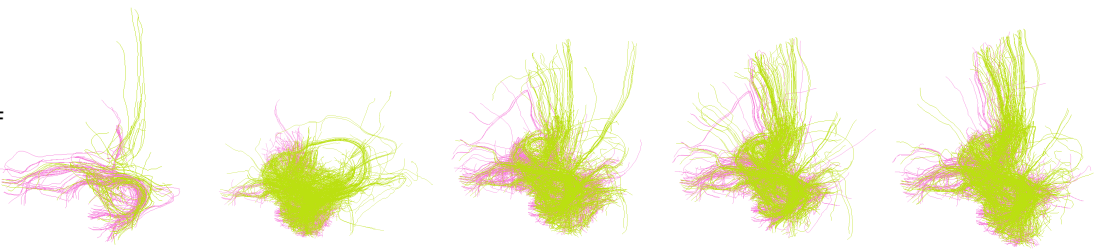

SLF
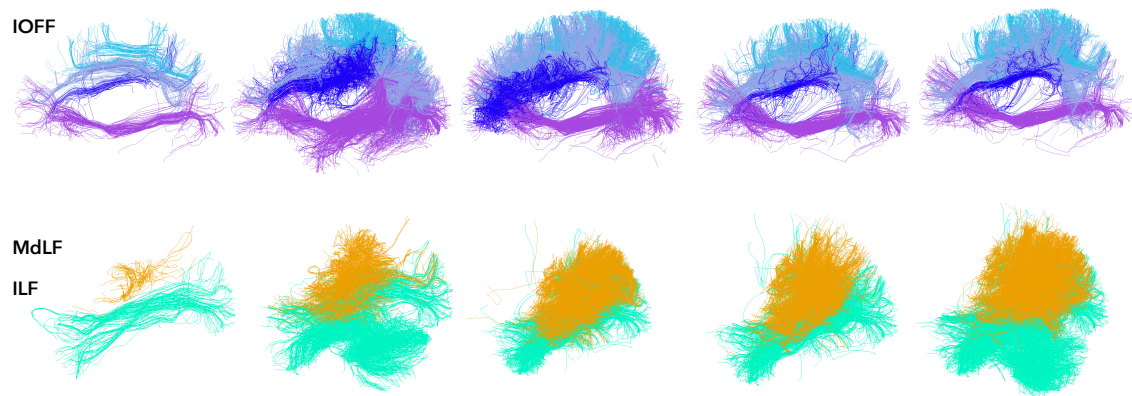

Fig. 3 Segmentation results on deterministic, whole brain tractography data, using the white matter query language (WMQL), k-means clustering, support vector classifiers (SVM) with linear and RBF-kernels, and random forests, shown for the corticospinal tract (CST), cingulum bundle (CB), corpus callosum (CC), actuate fasciculus (AF), unicate fasciculus (UF), superior, middle, and inferior longitudinal fasciculus (SLF, MdLF, ILF), and inferior occipitofrontal fasciculus (IOFF). 

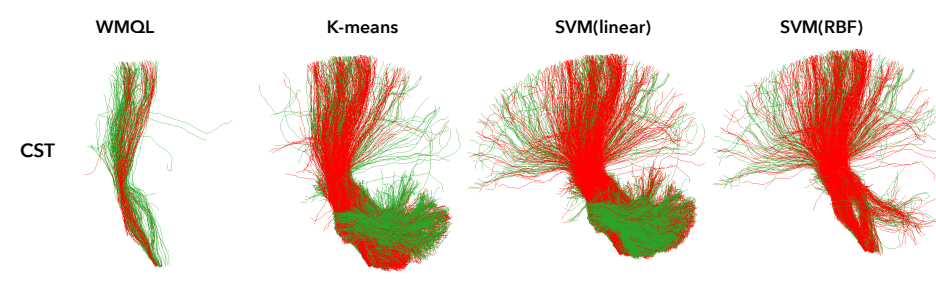

Random forests

CB
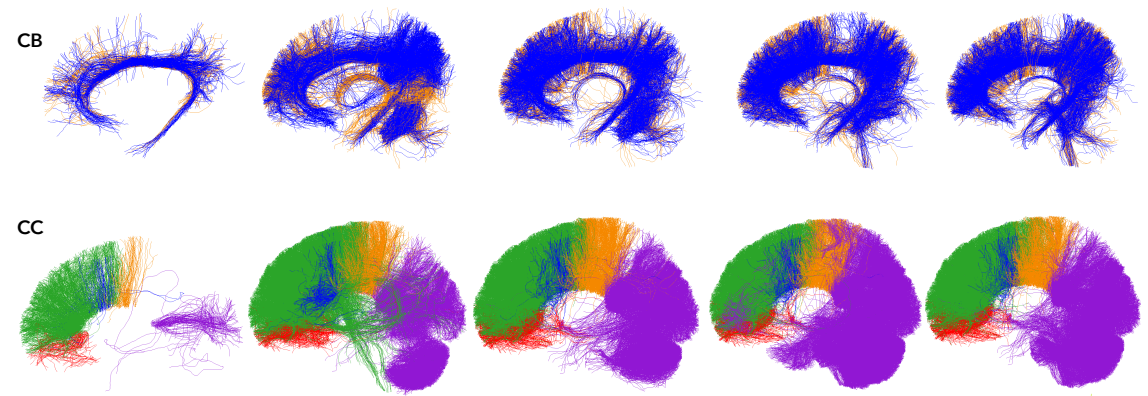

AF
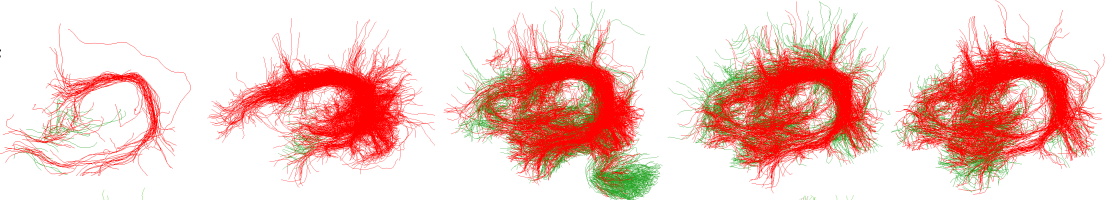

UF
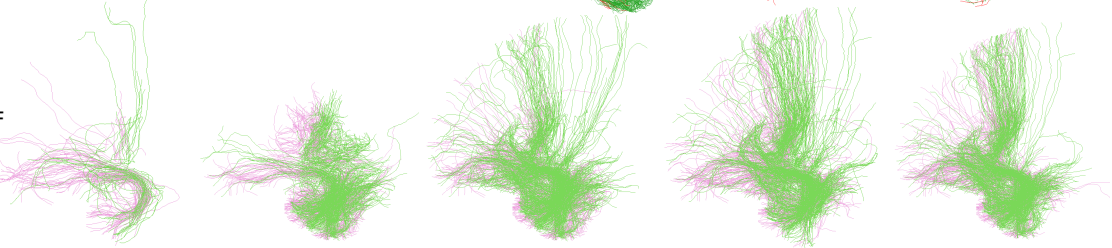

SLF
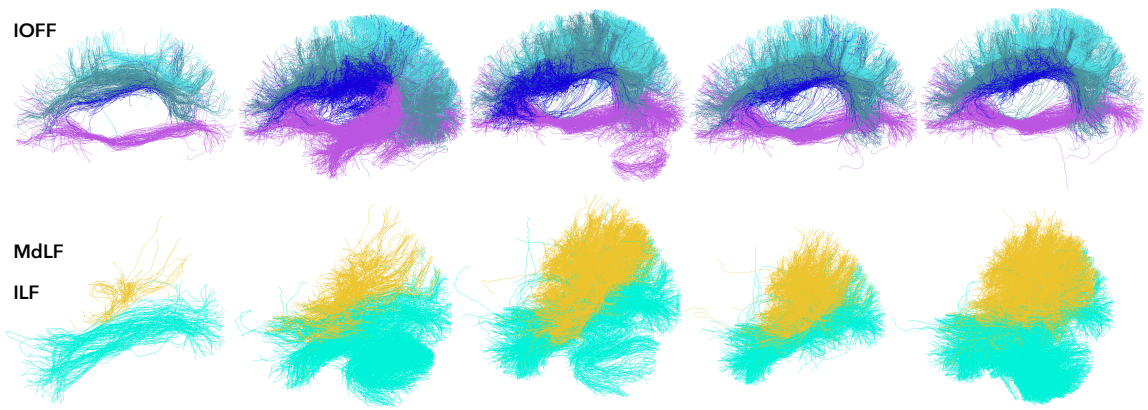

Fig. 4 Segmentation results on probabilistic, whole brain tractography data, using the white matter query language (WMQL), k-means clustering, support vector classifiers (SVM) with linear and RBF-kernels, and random forests, shown for the corticospinal tract (CST), cingulum bundle (CB), corpus callosum (CC), actuate fasciculus (AF), unicate fasciculus (UF), superior, middle, and inferior longitudinal fasciculus (SLF, MdLF, ILF), and inferior occipitofrontal fasciculus (IOFF). 


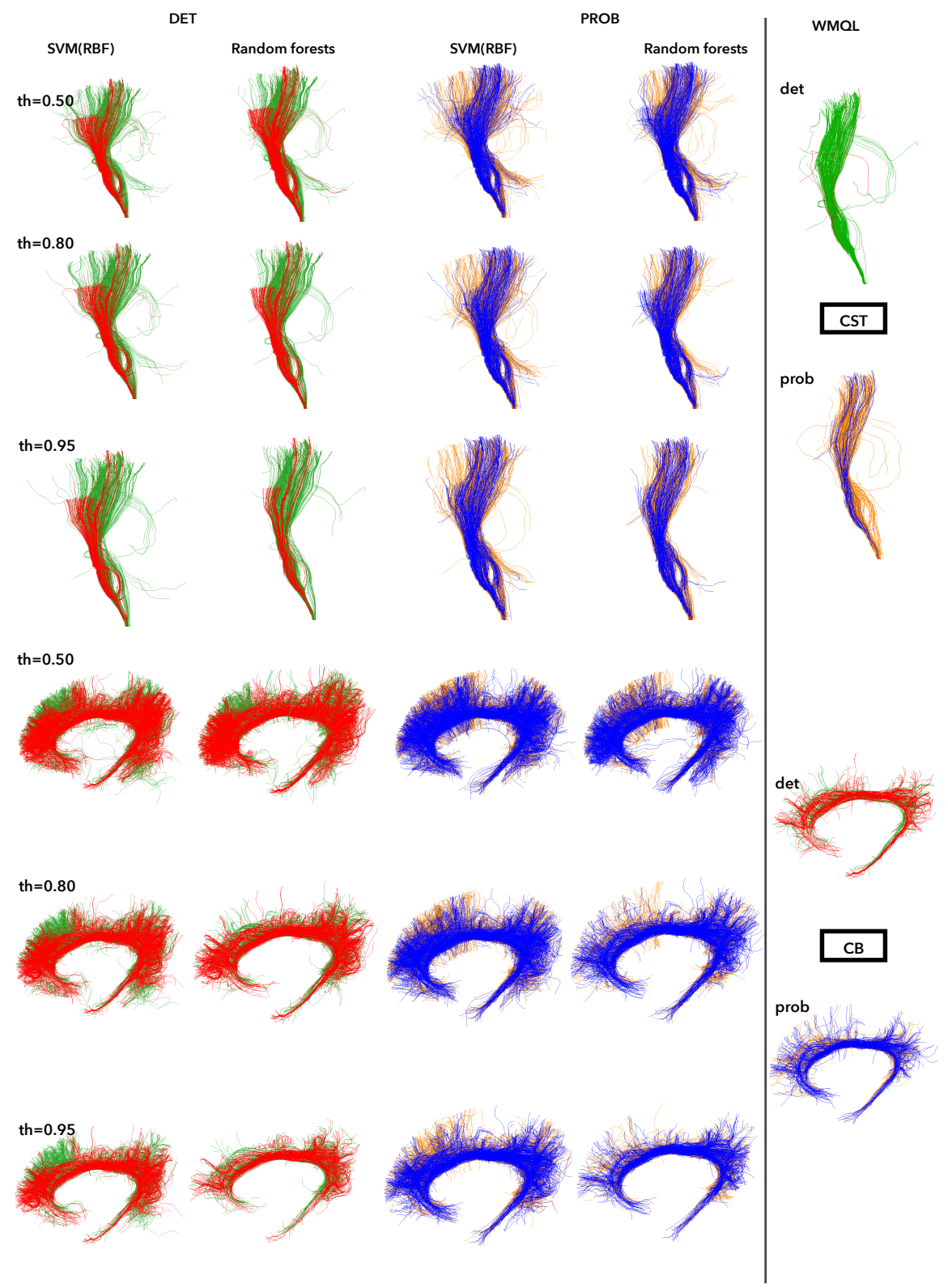

Fig. 5 Effect of different thresholds on the labeling probabilities for support vector machine (SVM) and random forest-based segmentation of the corticospinal tract (CST, top) and the cingulum bundle $(\mathrm{CB}$, bottom). The segmentation results of the white matter query language (WMQL) are shown for reference on the right. 
data (WMQL output). This can be explained by the fact that the WMQL excludes most outliers due to the strict requirements of the queries, resulting in a cleaner segmentation. As demonstrated, setting a threshold on the labeling probabilities creates a virtual outlier class that can be used to eliminate such tracks. Since setting the threshold too high can lead to sparse bundles, a method for optimal threshold selection still has to be developed.

Since there is no real ground truth available for white matter structures, it would be useful to let a neuroanatomy expert revise the segmentations obtained by the atlas. Expert knowledge could also be used to extend the WMQL with known bundles such as the fornix which is not yet included in the queries.

\section{Conclusion}

This work introduced a new method for fiber bundle segmentation, based on spectral embedding and supervised learning. The proposed method outperforms existing unsupervised methods and is more robust to outlier tracks when the label probability is taken into account. Future work may extend the atlas to smaller, currently undefined fiber bundles.

Acknowledgements Data were provided by the Human Connectome Project, WU-Minn Consortium (Principal Investigators: David Van Essen and Kamil Ugurbil; 1U54MH091657) funded by the 16 NIH Institutes and Centers that support the NIH Blueprint for Neuroscience Research; and by the McDonnell Center for Systems Neuroscience at Washington University.

\section{References}

1. Basser, P.J., Mattiello, J., LeBihan, D.: Mr diffusion tensor spectroscopy and imaging. Biophysical journal 66(1), 259-267 (1994)

2. Basser, P.J., Pajevic, S., Pierpaoli, C., Duda, J., Aldroubi, A.: In vivo fiber tractography using dt-mri data. Magnetic resonance in medicine 44(4), 625-632 (2000)

3. Beaulieu, C.: The basis of anisotropic water diffusion in the nervous system-a technical review. NMR in Biomedicine 15(7-8), 435-455 (2002)

4. Breiman, L.: Random forests. Machine learning 45(1), 5-32 (2001)

5. Conturo, T.E., Lori, N.F., Cull, T.S., Akbudak, E., Snyder, A.Z., Shimony, J.S., McKinstry, R.C., Burton, H., Raichle, M.E.: Tracking neuronal fiber pathways in the living human brain. Proceedings of the National Academy of Sciences 96(18), 10,422-10,427 (1999)

6. Cristianini, N., Shawe-Taylor, J.: An introduction to support vector machines and other kernelbased learning methods. Cambridge university press (2000)

7. Descoteaux, M., Deriche, R., Knosche, T., Anwander, A.: Deterministic and probabilistic tractography based on complex fibre orientation distributions. IEEE Transactions on Medical Imaging 28(2), 269-286 (2009)

8. Fowlkes, C., Belongie, S., Chung, F., Malik, J.: Spectral grouping using the nystrom method. Pattern Analysis and Machine Intelligence, IEEE Transactions on 26(2), 214-225 (2004) 
9. Glasser, M.F., Sotiropoulos, S.N., Wilson, J.A., Coalson, T.S., Fischl, B., Andersson, J.L., Xu, J., Jbabdi, S., Webster, M., Polimeni, J.R., Van Essen, D.C., Jenkinson, M.: The minimal preprocessing pipelines for the human connectome project. NeuroImage (2013)

10. Guevara, P., Duclap, D., Poupon, C., Marrakchi-Kacem, L., Fillard, P., Le Bihan, D., Leboyer, M., Houenou, J., Mangin, J.F.: Automatic fiber bundle segmentation in massive tractography datasets using a multi-subject bundle atlas. Neuroimage 61(4), 1083-1099 (2012)

11. Maddah, M., Grimson, W.E.L., Warfield, S.K., Wells, W.M.: A unified framework for clustering and quantitative analysis of white matter fiber tracts. Medical image analysis 12(2), 191-202 (2008)

12. Mori, S., van Zijl, P.: Fiber tracking: principles and strategies-a technical review. NMR in Biomedicine 15(7-8), 468-480 (2002)

13. O'Donnell, L.J., Golby, A.J., Westin, C.F.: Fiber clustering versus the parcellation-based connectome. NeuroImage 80, 283-289 (2013)

14. O'Donnell, L.J., Westin, C.F.: Automatic tractography segmentation using a high-dimensional white matter atlas. Medical Imaging, IEEE Transactions on 26(11), 1562-1575 (2007)

15. Parker, G.J.: Probabilistic fiber tracking. In: D.K. Jones (ed.) Diffusion MRI: Theory, methods, and applications, pp. 396-408. Oxford University Press (2010)

16. Shi, J., Malik, J.: Normalized cuts and image segmentation. Pattern Analysis and Machine Intelligence, IEEE Transactions on 22(8), 888-905 (2000)

17. Tournier, J., Calamante, F., Connelly, A.: Robust determination of the fibre orientation distribution in diffusion MRI: non-negativity constrained super-resolved spherical deconvolution. NeuroImage 35(4), 1459-1472 (2007)

18. Tournier, J.D., Mori, S., Leemans, A.: Diffusion tensor imaging and beyond. Magnetic Resonance in Medicine 65(6), 1532-1556 (2011)

19. Van Essen, D.C., Smith, S.M., Barch, D.M., Behrens, T.E., Yacoub, E., Ugurbil, K.: The WU-Minn human connectome project: an overview. NeuroImage 80, 62-79 (2013)

20. Vapnik, V.: The nature of statistical learning theory. Springer (2000)

21. Wassermann, D., Makris, N., Rathi, Y., Shenton, M., Kikinis, R., Kubicki, M., Westin, C.F.: On describing human white matter anatomy: The white matter query language. In: K. Mori, I. Sakuma, Y. Sato, C. Barillot, N. Navab (eds.) Medical Image Computing and ComputerAssisted Intervention-MICCAI 2013, Lecture Notes in Computer Science, vol. 8149, pp. 647-654. Springer Berlin Heidelberg (2013) 